\title{
Can An Algebraic Diagnostic Test be Used to Predict Final Grades in an Introductory Statistics Class?
}

\author{
Anthony Cooper, Justine Kirksey and Rafael E. Diaz* \\ Department of Mathematics and Statistics, California State University, Sacramento, CA
}

Students: aec97@csus.edu,jmk292@csus.edu

*Mentor:rdiaz@csus.edu

\begin{abstract}
The Department of Mathematics and Statistics at California State University Sacramento has been using the Intermediate Algebra Diagnostic (IAD) test as a proxy tool to screen students intending to enroll in an introductory statistics course (Stat 1). However, the use of an algebraic test as a diagnostic tool for a statistics course has been questioned by some faculty members and students at this university. The regression models used in this study (simple linear regression, hierarchical linear regression, and logistic regression) show that higher IAD scores are related to higher final grades in Stat 1, even after adjusting for different instructors. Inferences were also made in this study to predict a passing grade and passing rates in Stat 1 based on the bounds of the confidence and prediction intervals obtained for the IAD scores with these models.
\end{abstract}

\section{KEYWORDS}

Linear Regression, Logistic Regression, Statistics Diagnostic Test, Algebra Diagnostic Test, MDTP, Introductory Statistics

\section{BACKGROUND}

Past experience among faculty members in the Department of Mathematics and Statistics at California State University, Sacramento (CSUS) indicates the need to require some level of abstract reasoning and quantitative maturity from students registering in the course Introduction to Statistics (Stat 1). The department has never been able to find an appropriate existing diagnostic test for introductory statistics courses, so it has been using the Intermediate Algebra Diagnostic (IAD) test as a proxy tool to screen students intending to enroll in Stat 1. However, the use of an algebraic test as a diagnostic tool for a statistics course has been questioned by some faculty members and students at CSUS. Therefore there is a need for a study to determine if there is a relationship between the IAD scores and final grades in Stat 1. For this purpose the IAD scores and final grades in Stat 1 of over 550 students who have taken this course over a period of two semester terms and one summer session were collected to conduct such a study.

The IAD test is a product of the Mathematics Diagnostic Testing Project (MDTP) ${ }^{1}$. This project was founded in 1978 as a product of a joint endeavor between the University of California and the California State University systems, which aimed to design a measurement of student readiness for a wide range of mathematics courses. The original test was created not only by faculty members from these two university systems, but also by a workgroup of faculty from community colleges and high schools in California'. The current workgroup of developers of the test is assisted by the Educational Testing Service' ${ }^{1}$.

The IAD test and other tests created by the MDTP do not provide a scaling score (such as A, B, $\mathrm{C}$, etc.) since these tests are not intended to be used as final exams. Instead, raw scores (0 to 45 in the case of the IAD test) are reported to students to complement a placement procedure that should take into account previous coursework, grades, etc., as part of a reliable indicator of students' 
mathematical proficiency to succeed in a course ${ }^{1}$. Currently, the Mathematics and Statistics Department at CSUS requires a minimum IAD score of 32 for students to register in Stat 1 classes; however students with scores from 27-31 can sometimes register with a warning that they may have to work harder than the rest of the class to succeed in the course (the department calls this range of scores "Advisory Enrollment"). These are the same minimum scores required for Modern Business Math (Math 24), Calculus for Social and Life Sciences (Math 26A), and Precalculus (Math 29).

The literature on the Web page of the MDTP project states that in order to determine cutoff scores and "counseling ranges," statistical analyses of test scores and subsequent course performance should be conducted to validate the use of the test as a placement tool'. To date, no record of a statistical analysis of this kind exists for the Stat 1 course. However, the number of full time statisticians in the Mathematics and Statistics department at CSU Sacramento has increased from two to five in recent years, which has provided the department with the manpower needed to pay closer attention to this course, and to conduct such an analysis. The analysis presented here was conducted to investigate the question in the title of this article.

The Science Transfer Project (STP), which is part of the CSUS Science Educational Equity Program (SEE), aims to offer basic research experience to the underrepresented students that it serves. This project seeks to help students at the undergraduate level to learn, with faculty supervision, skills for carrying out elementary research in their fields of specialization. The analysis required for this study provides an opportunity that meets the goals of this program. The didactic style of this article stems from the need for the students involved in this project to both learn linear and logistic regression modeling while also learning how to analyze their results to test research hypotheses.

\section{METHODS}

The data collected for this analysis contains the IAD scores, final grades, and coded information of the Stat 1 classes for 564 students who took this course during the Summer and Fall of 2008, and the Spring of 2009. The original data set consisted of a larger number of students, but this number was reduced due to the inclusion criteria for this study, which excluded students who were taking this class for at least a second time and students who had taken more advanced mathematics classes prior to taking Stat 1.

Descriptive statistics of the data set are presented in Table 1 where a general increasing trend in final grades can be observed as the values of the measures of location statistics (mean, median and quartiles) for the IAD scores increase. 


\begin{tabular}{|c|c|c|c|c|c|c|c|c|}
\hline $\begin{array}{c}\text { Final } \\
\text { Grade }\end{array}$ & $\boldsymbol{n}(\%)$ & Min & $\boldsymbol{Q}_{1}$ & Median & $\boldsymbol{Q}_{3}$ & Max & $\overline{\boldsymbol{x}}$ & $\boldsymbol{S}$ \\
\hline A & $97(17)$ & 19 & 31 & 35 & 40 & 45 & 35.19 & 5.45 \\
\hline A- & $29(05)$ & 28 & 31 & 33 & 37 & 41 & 33.69 & 3.70 \\
\hline B+ & $93(16)$ & 22 & 30 & 32.5 & 35 & 41 & 32.52 & 4.00 \\
\hline B & $43(08)$ & 20 & 30 & 33 & 36 & 42 & 32.92 & 4.90 \\
\hline B- & $46(08)$ & 23 & 28 & 31 & 34 & 44 & 31.09 & 4.38 \\
\hline C+ & $86(15)$ & 26 & 28 & 31.5 & 34 & 42 & 31.64 & 4.21 \\
\hline C & $38(07)$ & 18 & 28 & 31 & 34 & 41 & 31.03 & 3.93 \\
\hline C- & $36(07)$ & 24 & 27 & 30.5 & 34 & 39 & 31.11 & 4.17 \\
\hline D+ & $36(07)$ & 23 & 31 & 32 & 34 & 36 & 31.57 & 4.20 \\
\hline D & $8(01)$ & 19 & 28 & 30 & 34 & 39 & 30.39 & 4.40 \\
\hline D- & $7(01)$ & 24 & 29.25 & 30.5 & 32.5 & 37 & 30.63 & 4.00 \\
\hline F & $45(08)$ & 19 & 27 & 30 & 33 & 44 & 30.47 & 4.77 \\
\hline
\end{tabular}

Table 1. Summary Statistics for the IAD scores by final grade: $n(\%)=$ number (percentage) of students who obtained the grade in column 1; Min = minimum IAD, Max = maximum IAD, $Q_{1}=25$ th percentile, Median, $Q_{3}=75$ th percentile, mean $\bar{x}$, and standard deviation $S$ of the IAD scores of students who obtained the grade in column 1.

The relationship between IAD scores and final grades was analyzed using linear and logistic regression techniques. The linear regression analysis allowed for inferences to be made regarding the minimum IAD scores required to pass STAT 1, while the logistic regression analysis allowed for inferences regarding probabilities of passing STAT 1 for each of the IAD scores observed.

For the linear regression analysis, final letter grades were converted to percentages in order to obtain a numerical response variable. Since each letter grade corresponds to an interval of percentages, two scales were obtained to reflect on the different criteria that can be used when assigning percentages to letter grades. Two regression models using each of these scales were fitted to the data, and inferences using confidence and prediction intervals from these models were subsequently compared. In addition, a hierarchical linear regression model was included to examine the hierarchical nature of the data (students nested within classrooms). For the logistic regression analysis, the grades were converted to a dichotomous outcome, pass or fail, using two criteria. In the first case, passing was defined as obtaining a grade of at least a D-, the general cutoff for a passing grade, while in the other passing was defined as obtaining a grade of at least $\mathrm{C}$-, which is the criterion used by many majors on campus that require Stat 1 .

The linear and logistic regression analyses were conducted using the statistical software $\mathrm{R}^{2}$, and the hierarchical linear regression model obtained using HLM $7^{3}$.

\section{ANALYSIS}

\subsection{Linear Regression Analysis}

The linear regression analysis is divided into three sections. In the first section two regression models are fitted using the two scales of percentages assigned to the final letter grades. In the second section these models are used to obtain confidence and prediction intervals for final percentage grades based on IAD scores. The third section presents an analysis using a hierarchical linear regression model to examine the hierarchical nature of the data where students are nested within classrooms. 


\subsubsection{Linear Regression Models}

When using the first scale, the numbers assigned to the final letter grades are the midpoints of the numerical intervals that are used to assign these letters based on the standard scale used at CSUS. For example, students who received an A as a final grade had an average final course percentage that fell into the range of 93 to 100; therefore, these students were assigned a numerical grade of 96.5 . For a grade of $\mathrm{F}$, which corresponds to a percentage from 0 to 59.9, a value of 57.95 was assigned to match the difference of 3.5 percentage points that exists between the midpoints for other letter grades. This numerical recoding of the final grades is listed in Table 2.

\begin{tabular}{|c|c|c|c|c|c|c|c|c|c|c|c|c|}
\hline $\begin{array}{c}\text { Letter } \\
\text { Grade }\end{array}$ & A & A- & B+ & B & B- & C+ & C & C- & D+ & D & D- & F \\
\hline $\begin{array}{c}\text { Numerical } \\
\text { Grade }\end{array}$ & 96.5 & 91.45 & 88.45 & 84.95 & 81.45 & 78.45 & 74.95 & 71.45 & 68.45 & 64.95 & 61.45 & 57.95 \\
\hline
\end{tabular}

Table 2. Numerical coding used for final letter grades (first scale).

In the scatterplot (shown with its least squares regression line) of numerical final scores against IAD scores in Figure 1, a positive linear trend is not very obvious to the naked eye due to the overlapping data points which appear in different shades. However, Figure 2 shows another graphical representation of this data set using boxplots of the numerical scores, without outliers, against each of the values of the IAD scores. In this plot it can be seen that for those students (a total of 41) who were admitted to a Stat 1 class by exception, with an IAD score below the minimum of 27 (see "Advisory Enrollment" in "Background"), the relationship of the IAD scores to the final numerical grades does not follow a linear trend. However, the median numerical final grades for each IAD score (represented by the segment in the middle of the boxplots) follows a general positive linear trend starting at about an IAD score of 26 and 27. Thus a linear regression model was fitted to the entire data set, and then separate linear regression models were fitted for each of the subsets of students with IAD scores below 27 and students with IAD scores of 27 and above. That is, the intercept $\beta_{0}$ and the slope $\beta_{1}$ of the linear equation of these data's numerical final scores, $y_{i}$, in terms of the IAD scores, $x_{i}$, are estimated via ordinary least squares regression:

$$
y_{i}=\beta_{0}+\beta_{1} x_{i}+e_{i} \quad \text { Equation } 1 .
$$

The $e_{i}$ in Equation 1 represent the effect of the particular set of students $i$ in this study who received a specific IAD score $x_{i}$. This model assumes $e_{i}$ to be normally distributed with mean zero and common variance for the numerical grades across groups of IAD scores.

It is helpful to scale the variable $x$ by subtracting the average of all IAD scores, $\bar{x}$, from the IAD score of each student, $\left(x_{i}-\bar{x}\right)$ (a process called "centering" in statistics) so that the intercept in Equation 1 matches the average of the numerical final grades for all students (the estimate of the slope does not change when centering the variable $x$ ):

$$
y_{i}=\beta_{0}+\beta_{1}\left(x_{i}-\bar{x}\right)+e_{i} \quad \text { Equation } 2 .
$$




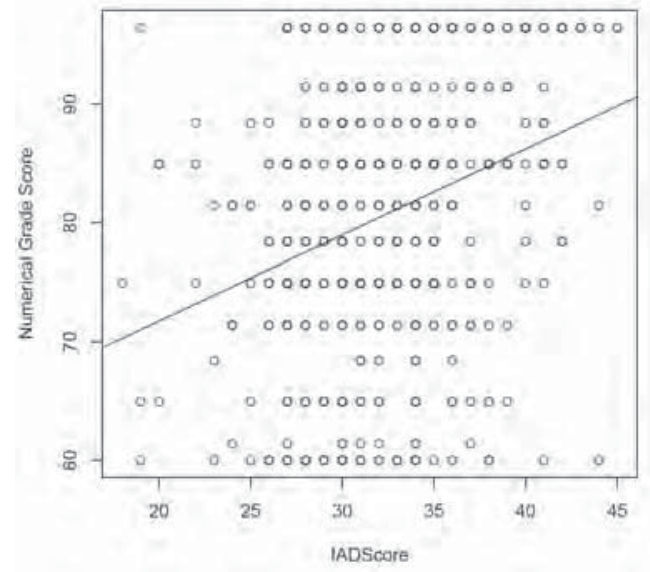

Figure 1. Numerical grade scores against IAD scores with least squares regression line (Scenario 1).

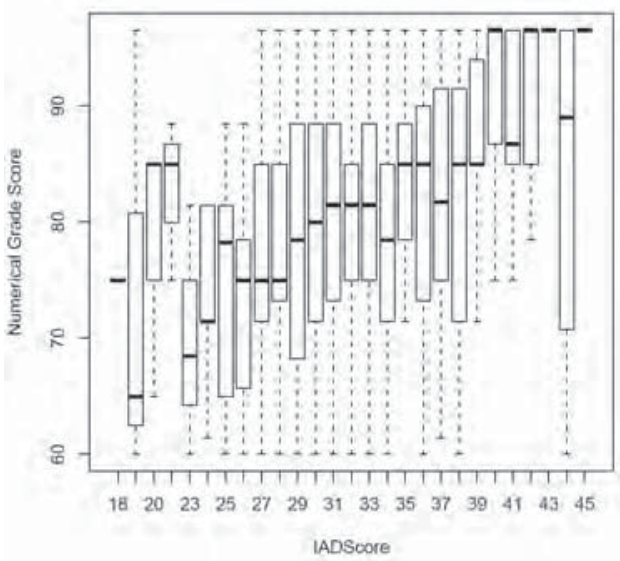

Figure 2. Boxplots of Numerical grade scores against IAD scores (first scale).

The estimated coefficients in Equation $\mathbf{2}$ when using the entire data set is:

$$
y_{i}=80.4727+0.7365\left(x_{i}-32.2814\right)+e_{i} \quad \text { Equation } 3 .
$$

Table 3 presents the summary of the estimates in Equation 3 where it can be seen that there is a significant statistical linear relationship (t-test, 562 d.f., p-value: $5.29 \times 10^{-14}$ ) between the IAD scores and the numerical version of the final grades. The slope in Equation 3 indicates that for each extra unit achieved in the IAD score, the estimated numerical final grade is expected to increase, on average, by about 0.74 of a percentage point. Of less importance, the p-value of the intercept of this centered equation is the $\mathrm{p}$-value for the t-test of the hypothesis that the average numerical final grades for the students in this data set, 80.4727 , is different from zero (however, it would be regrettable if this hypothesis were not rejected!). 


\begin{tabular}{|l|c|c|c|c|c|}
\hline & $\begin{array}{c}\text { Degrees of } \\
\text { Freedom }\end{array}$ & Estimate & $\begin{array}{c}\text { Standard } \\
\text { Error }\end{array}$ & t-value & $\operatorname{Pr}(>|\mathbf{t}|)$ \\
\hline Intercept & 1 & 80.4727 & 0.4616 & 174.34 & $<0.001$ \\
\hline Slope & 562 & 0.7365 & 0.0964 & 7.722 & $<0.001$ \\
\hline \multicolumn{6}{l}{ Residual Standard Error: 10.96 R-squared $=\mathbf{0 . 0 9 4 0 9}$} \\
\hline
\end{tabular}

Table 3. Summary for the regression model using the entire data set (first scale).

The estimates in Equation 3 changed only slightly when excluding the 41 students with IAD scores of 26 and below, and also when using students with IAD scores of 27 and above. Therefore, there was no need to provide separate analyses for these subgroups.

The second scale uses a conservative approach when assigning the numbers to the final letter grades: instead of using the midpoints of the numerical intervals, mentioned above, that correspond to these letters, it uses their lower bounds, with the exception of using 50 for the interval [0-59] that corresponds to the letter F. This numerical coding of the letter grades is shown in Table 4.

\begin{tabular}{|c|c|c|c|c|c|c|c|c|c|c|c|c|}
\hline $\begin{array}{c}\text { Letter } \\
\text { Grade }\end{array}$ & $\mathrm{A}$ & $\mathrm{A}-$ & $\mathrm{B}+$ & $\mathrm{B}$ & $\mathrm{B}-$ & $\mathrm{C}+$ & $\mathrm{C}$ & $\mathrm{C}-$ & $\mathrm{D}+$ & $\mathrm{D}$ & $\mathrm{D}-$ & $\mathrm{F}$ \\
\hline $\begin{array}{c}\text { Numerical } \\
\text { Grade }\end{array}$ & 93 & 90 & 87 & 83 & 80 & 77 & 73 & 70 & 67 & 63 & 60 & 50 \\
\hline
\end{tabular}

Table 4. Numerical coding used for final letter grades (second scale).

The estimates and summary of the regression equation when using the numerical final grades in Table 4 are shown in Equation 4 and Table 5, respectively. It is no surprise that the slope of Equation 4 is almost the same as that of Equation $\mathbf{3}$ since changing the numerical grades from the first to the second scale amounts to an almost vertical downward shift (numerical grades were decreased by almost the same constant amount) in the data points. The intercept in Equation $\mathbf{4}$ does reflect this downward shift in predicted final grades sought with this model.

$$
y_{i}=77.9592+0.7371\left(x_{i}-32.9446\right)+e_{i} \quad \text { Equation } 4 .
$$

\begin{tabular}{|l|c|c|c|c|c|}
\hline & $\begin{array}{c}\text { Degrees of } \\
\text { Freedom }\end{array}$ & Estimate & $\begin{array}{c}\text { Standard } \\
\text { Error }\end{array}$ & t-value & $\operatorname{Pr}(>|\mathbf{t}|)$ \\
\hline Intercept & 1 & 77.9592 & 0.4917 & 158.548 & $<0.001$ \\
\hline Slope & 562 & 0.7371 & 0.1027 & 7.177 & $<0.001$ \\
\hline \multicolumn{7}{l}{ Residual Standard Error: 11.68 R-squared $=\mathbf{0 . 0 8 3 9 7}$} \\
\hline
\end{tabular}

Table 5. Summary for the regression equation using students with IAD scores of 27 and above (second scale). 


\subsubsection{Confidence Intervals (CI) and Prediction Intervals (PI)}

The least squares regression lines provide estimates for the population average numerical final grade for each of the IAD scores. Upper and lower bounds for $95 \%$ confidence intervals for the average numerical final grade of students broken down by IAD score are listed in Tables 6 and 7 for models (3) and (4), respectively. These bounds were computed using the formula in Equation $5^{4}$. Assuming the grades and scores of the students included in this study are a representative sample of those of the population of students who take Stat 1, one can be $95 \%$ confident that the average numerical final numerical grade in Stat 1 of the population of students with a given IAD score falls between these two bounds. Note that, under both models, one can be $95 \%$ confident that even students with an IAD score of 18 will get, on average, a final grade of at least $64.3953 \%$, which corresponds to at least a grade of D. On the other hand, students with an IAD score of at least 22 under model (3), and at least 25 under model (4), can be 95\% confident to obtain, on average, a final numerical grade above the minimum numerical final grade of $70 \%$ (corresponding to a grade of $\mathrm{C}-$ ) required as a passing grade by most majors on campus that have this class as a requirement.

$$
\bar{y}+\hat{\beta}_{1}\left(x_{0}-\bar{x}\right) \pm t_{n-2,1-\frac{\alpha}{2}} S_{y \mid x} \sqrt{\frac{1}{n}+\frac{\left(x_{0}-\bar{x}\right)^{2}}{(n-1) s_{x}^{2}}} \quad \text { Equation } 5
$$

\begin{tabular}{|c|l|l|l|l|c|l|l|l|l|}
\hline IAD & CI:Lower & CI:Upper & PI:Lower & PI:Upper & IAD & CI:Lower & CI:Upper & PI:Lower & PI:Upper \\
\hline 18 & 67.10279 & 72.80611 & 48.23899 & 91.6699 & 32 & 79.35742 & 81.17348 & 58.71891 & $100^{*}$ \\
\hline 19 & 68.01811 & 73.36379 & 48.99825 & 92.38365 & 33 & 80.08533 & 81.91857 & 59.45504 & $100^{*}$ \\
\hline 20 & 68.93188 & 73.92301 & 49.75588 & 93.09902 & 34 & 80.77536 & 82.70154 & 60.18951 & $100^{*}$ \\
\hline 21 & 69.84375 & 74.48414 & 50.51187 & 93.81602 & 35 & 81.43256 & 83.51734 & 60.92232 & $100^{*}$ \\
\hline 22 & 70.75325 & 75.04764 & 51.26623 & 94.53467 & 36 & 82.06371 & 84.35919 & 61.65347 & $100^{*}$ \\
\hline 23 & 71.65976 & 75.61414 & 52.01894 & 95.25496 & 37 & 82.67527 & 85.22063 & 62.38296 & $100^{*}$ \\
\hline 24 & 72.56243 & 76.18447 & 52.77 & 95.97689 & 38 & 83.27243 & 86.09647 & 63.11079 & $100^{*}$ \\
\hline 25 & 73.4601 & 76.75979 & 53.51942 & 96.70048 & 39 & 83.85903 & 86.98286 & 63.83696 & $100^{*}$ \\
\hline 26 & 74.35117 & 77.34173 & 54.26718 & 97.42572 & 40 & 84.43784 & 87.87705 & 64.56148 & $100^{*}$ \\
\hline 27 & 75.23334 & 77.93255 & 55.01328 & 98.15261 & 41 & 85.01082 & 88.77708 & 65.28434 & $100^{*}$ \\
\hline 28 & 76.10344 & 78.53546 & 55.75773 & 98.88117 & 42 & 85.57935 & 89.68155 & 66.00555 & $100^{*}$ \\
\hline 29 & 76.95703 & 79.15486 & 56.50052 & 99.61138 & 43 & 86.14444 & 90.58945 & 66.72512 & $100^{*}$ \\
\hline 30 & 77.78834 & 79.79656 & 57.24164 & $100.000^{*}$ & 44 & 86.70684 & 91.50006 & 67.44305 & $100^{*}$ \\
\hline 31 & 78.59058 & 80.46732 & 57.98111 & $100.000^{*}$ & 45 & 87.26709 & 92.41281 & 68.15934 & $100^{*}$ \\
\hline
\end{tabular}

Table 6. Confidence Intervals (CI) and Prediction Intervals (PI) for IAD scores (first scale).

${ }^{*}$ Indicates truncated bounds due to overshoot.

Of more interest for this study is the construction of prediction intervals, which differ in a fundamental way from confidence intervals: prediction intervals provide an interval estimate for a future individual numerical final grade of a student who enrolls in Stat 1 with a particular IAD score, rather than the average numerical final grade for a population of individuals who have enrolled in a Stat 1 course and who share a particular common IAD score. The lower and upper bounds for this kind of interval, broken down by IAD score, were obtained using Equation $6^{4}$ for each of the models. The bounds of these intervals are also listed in Tables $\mathbf{6}$ and $\mathbf{7}$.

$$
\bar{y}+\hat{\beta}_{1}\left(x_{0}-\bar{x}\right) \pm t_{n-2,1-\frac{\alpha}{2}} S_{y \mid x} \sqrt{\frac{1}{n}+\frac{\left(x_{0}-\bar{x}\right)^{2}}{(n-1) s_{x}^{2}}}
$$

Equation 6. 


\begin{tabular}{|c|l|l|l|l|c|l|l|l|l|}
\hline IAD & CI:Lower & CI:Upper & PI:Lower & PI:Upper & IAD & CI:Lower & CI:Upper & PI:Lower & PI:Upper \\
\hline 18 & 64.3953 & 70.4715 & 44.29639 & 90.57041 & 32 & 76.78527 & 78.72019 & 54.79566 & $100^{*}$ \\
\hline 19 & 65.32292 & 71.01807 & 45.05773 & 91.28326 & 33 & 77.51316 & 79.46649 & 55.53237 & $100^{*}$ \\
\hline 20 & 66.24889 & 71.56629 & 45.81734 & 91.99784 & 34 & 78.20069 & 80.25314 & 56.26730 & $100^{*}$ \\
\hline 21 & 67.17283 & 72.11654 & 46.5752 & 92.71417 & 35 & 78.85326 & 81.07477 & 57.00046 & $100^{*}$ \\
\hline 22 & 68.09425 & 72.66931 & 47.33132 & 93.43224 & 36 & 79.47808 & 81.92414 & 57.73185 & $100^{*}$ \\
\hline 23 & 69.01248 & 73.22527 & 48.08568 & 94.15207 & 37 & 80.08203 & 82.79438 & 58.46147 & $100^{*}$ \\
\hline 24 & 69.92661 & 73.78533 & 48.83829 & 94.87365 & 38 & 80.67065 & 83.67995 & 59.18932 & $100^{*}$ \\
\hline 25 & 70.83543 & 74.35070 & 49.58915 & 95.59698 & 39 & 81.24802 & 84.57676 & 59.91541 & $100^{*}$ \\
\hline 26 & 71.73719 & 74.92313 & 50.33824 & 96.32208 & 40 & 81.81709 & 85.48188 & 60.63974 & $100^{*}$ \\
\hline 27 & 72.62949 & 75.50502 & 51.08556 & 97.04895 & 41 & 82.37995 & 86.39321 & 61.36230 & $100^{*}$ \\
\hline 28 & 73.50890 & 76.09980 & 51.83112 & 97.77758 & 42 & 82.93808 & 87.30928 & 62.08311 & $100^{*}$ \\
\hline 29 & 74.37073 & 76.71216 & 52.57491 & 98.50798 & 43 & 83.49254 & 88.229 & 62.80216 & $100^{*}$ \\
\hline 30 & 75.20881 & 77.34827 & 53.31693 & 99.24014 & 44 & 84.04413 & 89.1516 & 63.51947 & $100^{*}$ \\
\hline 31 & 76.01590 & 78.01537 & 54.05718 & 99.97409 & 45 & 84.59344 & 90.07649 & 64.23503 & $100^{*}$ \\
\hline
\end{tabular}

Table 7. Confidence Intervals (CI) and Prediction Intervals (PI) for IAD scores (second scale).

${ }^{*}$ Indicates truncated bounds due to overshoot.

Note that these intervals, on the one hand, indicate that for a randomly chosen student with a grade of at least 34 under model (3), and a at least 40 under model (4), one can be $95 \%$ confident that he/she will pass Stat 1 with a numerical final grade of at least $60 \%$, which corresponds to a grade of at least a D-. On the other hand, one could argue that this model also predicts that if this student has an IAD score as low as 18, his/her predicted final grade may be as high an A- (91.6\%) - the same can be said with for the model that uses the more conservative scale, although with a lower percentage final grade that still corresponds to an A- (90.57\%). Note also that the prediction intervals of IAD scores as low as 30 of this model have upper bounds that needed to be truncated due to overshot (upper bounds above the maximum of 100\%).

It can be then concluded then, that the variation in final grades for each IAD score did not allow the regression models to provide prediction or confidence intervals tight enough to predict accurately, final grades in terms of IAD scores.

\subsubsection{Hierarchical Linear and Logistic Regression Models (Adjusting the Model for Classrooms)}

The data set of this study consists of students from 32 classes taught by a total of 11 instructors. The linear regression models above assume that the effect of IAD scores is the same across classrooms and instructors. However, it is possible that the relationship between IAD scores and final grades varies by instructor, and even by class taught by the same instructor. Instructors may have different styles or abilities, and dynamics between students and instructor may also differ from class to class. The levels of the variable instructor (that is, the instructors themselves) are not fixed categories of individual interest in this analysis. Therefore, instead of coding the instructors using dummy variables ( 10 of them would be needed given, that there are 11 instructors), the effect of this relatively large number of instructors can be modeled using random coefficients in a hierarchical linear model $^{5}$.

In order to introduce the idea of a hierarchical linear model, one can consider first the not so practical alternative of fitting a regression line for each of the 32 classes in this data set. Thus, if two of these lines were to be compared, the line with the higher intercept would be indicative of a higher numerical final grade for the corresponding class. Also, if both lines had a positive slope, the line 
with the larger slope would be indicative of a stronger effect of IAD scores on final grades. Now out of all of these 32 lines, lines with the largest intercepts and smallest slopes (high final grades that do not depend much on IAD scores) could be indicative of a lenient instructor, or also of a very effective instructor whose students' high grades do not depend much on IAD scores. Further interpretations could be drawn from other combinations of intercepts' and slopes' relative magnitudes.

Given the impracticality of fitting a line for each class, a hierarchical linear model could be more effective in this situation. A simple model of this kind assumes that each classroom $j$ (level-2 unit) is a random sample from a population of classrooms whose effects $U_{0 j}$ on final grades, after adjusting for the effect of IAD scores, are random variables with mean zero and common variance $\tau_{0}^{2}$ (population between-group variance). The model also assumes that each student $i$ (level-1 unit) within a classroom $j$ is a random sample from a population of students whose effects $e_{i j}$ on final grades, after adjusting for the effect of IAD scores, are random variables with mean zero and common variance (population within-group variance) $\sigma^{2}$ within each classroom. A final assumption linking these variance components is that the random effects $U_{0 j}$ are independent of the random effects $e_{i j}$. Thus the final grades of a student $i$ in classroom $j, y_{i j}$, can be represented linearly in terms of these random effects and the student's cluster-centered IAD score $\left(x_{i j}-\bar{x}_{i j}\right)$ as described in the following equation:

$$
y_{i j}=\gamma_{00}+\gamma_{10}\left(x_{i j}-\bar{x}_{j j}\right)+U_{0 j}+e_{i j}
$$

Equation 7.

In terms of the notation used by Snijders and Bosker ${ }^{5}$ this equation can be seen as a model that allows for classroom-specific regression models $y_{i j}=\beta_{0}+\beta_{1}\left(x_{i j}-\bar{x}_{j}\right)+e_{i j}$ that share the same slope, $\left(\beta_{1}=\gamma_{10}\right)$, but permits separate random intercepts for each classroom $j\left(\beta_{0}=\gamma_{00}+U_{0 j}\right)$. As the reader may have surmised, in order to allow for these models to also have classroom-specific slopes, one would need to add a third set of random effects $U_{1 j}\left(\beta_{1}=\gamma_{10}+U_{1 j}\right)$ for each classroom $j$, which would be independent of the other two sets of random effects, and would also be assumed to be random variables with mean zero and variance $\tau_{1}^{2}$. The resulting model would be:

$$
y_{i j}=\left(\gamma_{00}+U_{0 j}\right)+\left(\gamma_{10}+U_{1 j}\right)\left(x_{i j}-\bar{x}_{. j}\right)+e_{i j} \quad \text { Equation } 8 .
$$

Table 8 below presents the summary of model (7) when estimating its parameters with the statistical software HLM7. A p-value of the t-test less than 0.001 for the variance component $\tau_{0}^{2}$ of the random intercept indicates that the classrooms do differ in their average final grades. The coefficients for the fixed effects of the intercept and the slope are very close to those obtained with the simple regression model (see Table 3), which does not account for the clustering effect of the classrooms; however, the standard error for the intercept of the hierarchical model (0.778995) is almost twice that of the simple regression model $(0.4670)$. This is an indication that these two estimates are rather different from a statistical point of view. In the words of Snijder and Bosker5, the simple regression model (3) "produces an over-optimistic impression of the precision of this estimate, and illustrates the lack of trustworthiness of using simple regression modeling for estimates of multilevel data." Rounding the estimates in Table 8 to two decimals, a low average final grade classroom (bottom 2.5 percent) has a value of $U_{0 j}$ of about 2 standard deviations below the mean, so that it will have a regression line:

$$
y_{i j}=80.83-2(0.78)+0.72 I A D=79.27+0.72 I A D \quad \text { Equation } 9 .
$$

This regression line differs slightly from that of a typical high average final grade classroom (upper 2.5 percent):

$$
y_{i j}=80.83-2(0.78)+0.72 I A D=82.39+0.72 I A D \quad \text { Equation } 10 .
$$

\section{$A \prod R$ Volume 12 | Issue 2 | January 2015}




\begin{tabular}{|c|c|c|c|c|c|}
\hline Fixed Effect & $\begin{array}{c}\text { Degrees of } \\
\text { Freedom }\end{array}$ & Coefficient & $\begin{array}{c}\text { Standard } \\
\text { Error }\end{array}$ & t-value & $\operatorname{Pr}(>|\mathbf{t}|)$ \\
\hline Intercept $\gamma_{0}$ & 31 & 80.829332 & 0.778995 & 103.7611 & $<0.001$ \\
\hline Slope $\gamma_{1}$ & 490 & 0.719604 & 0.11902 & 6.431 & $<0.001$ \\
\hline Random Effect & $\begin{array}{c}\text { Degrees of } \\
\text { Freedom }\end{array}$ & $\begin{array}{c}\text { Estimate of } \\
\text { Variance } \\
\text { Component }\end{array}$ & $\begin{array}{c}\text { Estimate of } \\
\text { Standard } \\
\text { Deviation } \tau_{0}\end{array}$ & $\begin{array}{c}\text { Chi } \\
\text { Square }\end{array}$ & p-value \\
\hline Var of $U_{0 j}\left(\tau_{0}^{2}\right)$ & 31 & 12.18985 & 3.49149 & 86.97264 & $<0.001$ \\
\hline Var of $e_{i j}\left(\sigma^{2}\right)$ & & 110.86590 & 10.52929 & & \\
\hline
\end{tabular}

Table 8. Summary for the hierarchical regression model in Equation 7.

The difference between the intercepts of lines (9) and (10), although statistically different, presents no practical difference, since the intercepts of these two lines differ (point estimates for the population average numerical final scores of classes in the 2.5th and 97.5th percentiles, respectively) by barely half a letter grade.

The hierarchical regression model (8) that allows for different slopes $\left(\gamma_{10}+U_{1 j}\right)$ produced no statistical difference between the slopes of the regression lines of the classrooms ( $\mathrm{p}$-value of $\mathrm{t}$-test for the null hypothesis, $\operatorname{var}\left(U_{0 j}\right)=\tau_{1}^{2}=0$, greater than 0.5 ) as shown in the summary of this model in Table 9.

\begin{tabular}{|c|c|c|c|c|c|}
\hline Fixed Effect & $\begin{array}{c}\text { Degrees of } \\
\text { Freedom }\end{array}$ & Coefficient & $\begin{array}{c}\text { Standard } \\
\text { Error }\end{array}$ & t-value & $\operatorname{Pr}(>|\mathbf{t}|)$ \\
\hline Intercept $\gamma_{0}$ & 31 & 80.838120 & 0.779788 & 103.667 & $<0.001$ \\
\hline Slope $\gamma_{1}$ & 490 & 0.726215 & 0.114033 & 6.368 & $<0.001$ \\
\hline Random Effect & $\begin{array}{c}\text { Degrees of } \\
\text { Freedom }\end{array}$ & $\begin{array}{c}\text { Estimate of } \\
\text { Variance } \\
\text { Component }\end{array}$ & $\begin{array}{c}\text { Estimate of } \\
\text { Standard } \\
\text { Deviation } \tau_{0}\end{array}$ & $\begin{array}{c}\text { Chi } \\
\text { Square }\end{array}$ & p-value \\
\hline Var of $U_{0 j}\left(\tau_{0}^{2}\right)$ & 31 & 12.2468 & 3.49954 & 87.14417 & $<0.001$ \\
\hline Var of $U_{1 j}\left(\tau_{1}^{2}\right)$ & 31 & 0.01423 & 0.11928 & 29.16380 & $>0.5$ \\
\hline Var of $e_{i j}\left(\sigma^{2}\right)$ & & 110.64026 & 10.51857 & & \\
\hline
\end{tabular}

Table 9. Summary for the hierarchical regression model in Equation 8. 


\subsection{Logistic Regression Analysis}

As an alternative to converting final letter grades to approximate percentages, a logistic regression equation can be obtained to model the probability of passing the course using IAD scores as a predictor variable. This requires recoding the passing grades with a 1 and the failing grades with a 0 . If the probability of passing the course given an IAD score $x_{i}$ is denoted as $\pi\left(x_{i}\right)$, the logodds (logarithm of the odds) or logit of these probabilities, $\operatorname{logit}\left[\pi\left(x_{i}\right)\right]=\log \left[\pi\left(x_{i}\right)\left(1-\pi\left(x_{i}\right)\right)\right]$, can be predicted as a linear function of the IAD scores.

If passing the course is defined as obtaining a grade of at least $\mathrm{D}$-, the logistic regression equation when using the entire data set is given by:

$$
\log \left(\frac{\pi\left(x_{i}\right)}{1-\pi\left(x_{i}\right)}\right)=-0.37117+0.08958 x_{i} \quad \text { Equation } 11 .
$$

Whereas if passing the course is defined of obtaining a grade of at least a C-, the equation is:

$$
\log \left(\frac{\pi\left(x_{i}\right)}{1-\pi\left(x_{i}\right)}\right)=-1.48029+0.09706 x_{i} \quad \text { Equation } 12
$$

The summaries of models (11) and (12), listed in Tables 10 and 11, respectively, indicate a significant relationship between the IAD scores and the probability of passing the course (p-values of $z$-tests for slopes less than 0.01). However, the null hypothesis that the intercept is zero cannot be rejected in both of the models. This lack of significance for the intercepts has no intrinsic value in this analysis since there are no students who enroll in the course with an IAD score of zero. The Chi-square tests with 1 degree of freedom (d.f.) at the bottom of these tables are goodness of fit tests. For both models, this test rejects the null hypothesis that the fit of the model that uses the IAD as a predictor is no better than the model with just an intercept (null model).

\begin{tabular}{|l|c|c|c|c|}
\hline & Estimate & Standard Error & z-value & $\operatorname{Pr}(>|\mathbf{z}|)$ \\
\hline Intercept & -0.37117 & 1.05491 & -0.352 & 0.72495 \\
\hline Slope & 0.08958 & 0.03405 & 2.631 & $<0.01$ \\
\hline Chi-square $=$ 7.2, d.f. $=\mathbf{1}, \mathbf{p}<\mathbf{0 . 0 1}$ \\
\hline
\end{tabular}

Table 10. Summary for the logistic regression Equation 11 if passing grade is at least D-.

\begin{tabular}{|l|c|c|c|c|}
\hline & Estimate & Standard Error & z-value & $\operatorname{Pr}(>|\mathbf{z}|)$ \\
\hline Intercept & -1.48029 & .078449 & -1.887 & 0.059168 \\
\hline Slope & 0.09706 & 0.02511 & 3.865 & $<0.001$ \\
\hline Chi-square $=\mathbf{1 5 . 9 1}$, d.f. $=\mathbf{1}, \mathbf{p}<\mathbf{0 . 0 0 0 1}$ \\
\hline
\end{tabular}

Table 11. Summary for the logistic regression Equation 12 if passing grade is at least C-.

When solving for the predicted probabilities $\pi\left(x_{i}\right)$ in terms of the intercept $\beta_{0}$, and the slope $\beta_{1}$ in models (11) and (12), the resulting equation is:

$$
\pi\left(x_{i}\right)=\left(\frac{\exp \left(\beta_{0}+\beta_{1} x_{i}\right)}{1+\exp \left(\beta_{0}+\beta_{1} x_{i}\right)}\right) \quad \text { Equation } 13 .
$$


Tables 12 and 13 list the predicted probabilities, for the given IAD scores, of passing the course with a D- and a C-, respectively. These tables also list the 95\% confidence intervals of these probabilities.

\begin{tabular}{|c|c|c|c|c|c|c|c|}
\hline IAD & Probability & CI: Lower & CI:Upper & IAD & Probability & CI: Lower & CI:Upper \\
\hline 18 & 0.7757727 & 0.5850923 & 0.8946066 & 32 & 0.923809 & 0.8980247 & 0.9434841 \\
\hline 19 & 0.7909688 & 0.6214484 & 0.8971406 & 33 & 0.9298794 & 0.9037926 & 0.9492896 \\
\hline 20 & 0.8053934 & 0.6563261 & 0.8996857 & 34 & 0.9354999 & 0.9084203 & 0.9549692 \\
\hline 21 & 0.8190504 & 0.6894004 & 0.9022551 & 35 & 0.9406986 & 0.9122341 & 0.9603329 \\
\hline 22 & 0.8319489 & 0.7204120 & 0.9048659 & 36 & 0.9455028 & 0.9154722 & 0.965269 \\
\hline 23 & 0.844103 & 0.7491678 & 0.9075414 & 37 & 0.9499384 & 0.9182990 & 0.9697291 \\
\hline 24 & 0.8555307 & 0.7755373 & 0.9103131 & 38 & 0.9540305 & 0.9208251 & 0.9737075 \\
\hline 25 & 0.8662534 & 0.7994422 & 0.9132239 & 39 & 0.9578031 & 0.9231254 & 0.9772238 \\
\hline 26 & 0.8762954 & 0.8208449 & 0.9163327 & 40 & 0.9612785 & 0.9252513 & 0.980311 \\
\hline 27 & 0.8856829 & 0.8397333 & 0.9197179 & 41 & 0.9644784 & 0.9272387 & 0.9830079 \\
\hline 28 & 0.8944438 & 0.8561118 & 0.9234769 & 42 & 0.9674228 & 0.9291132 & 0.9853549 \\
\hline 29 & 0.9026072 & 0.8700039 & 0.9277129 & 43 & 0.9701307 & 0.9308935 & 0.9873916 \\
\hline 30 & 0.9102026 & 0.8814821 & 0.9324961 & 44 & 0.9726198 & 0.9325935 & 0.9891547 \\
\hline 31 & 0.9172599 & 0.8907203 & 0.9378042 & 45 & 0.9749069 & 0.9342239 & 0.9906783 \\
\hline
\end{tabular}

Table 12. Predicted Probabilities and Confidence Intervals for passing with grade of at least D-.

The confidence interval in Table $\mathbf{1 2}$ for the current minimum required IAD score of 32 indicates that one can be $95 \%$ confident that at the most $10 \%(1-0.898=0.102)$ of students with and IAD score of 32 are expected to fail the class with a grade of $\mathrm{F}$. T-0.802 $=0.198$ ) if a $\mathrm{C}$ - is required to pass the course (see Table 13).

\begin{tabular}{|c|c|c|c|c|c|c|c|}
\hline IAD & Probability & CI: Lower & CI:Upper & IAD & Probability & CI: Lower & CI:Upper \\
\hline 18 & 0.5663090 & 0.3997442 & 0.7191292 & 32 & 0.8355744 & 0.8016251 & 0.8646944 \\
\hline 19 & 0.5899766 & 0.4345689 & 0.7292797 & 33 & 0.8484791 & 0.8141652 & 0.8774111 \\
\hline 20 & 0.6132346 & 0.4699295 & 0.7392909 & 34 & 0.8605401 & 0.8249142 & 0.8898846 \\
\hline 21 & 0.6359874 & 0.5054408 & 0.749175 & 35 & 0.8717861 & 0.8343183 & 0.9017783 \\
\hline 22 & 0.6581479 & 0.5407022 & 0.7589502 & 36 & 0.8822493 & 0.8427208 & 0.9128704 \\
\hline 23 & 0.6796388 & 0.5753120 & 0.7686434 & 37 & 0.8919644 & 0.8503639 & 0.9230462 \\
\hline 24 & 0.7003937 & 0.6088788 & 0.7782927 & 38 & 0.9009680 & 0.8574136 & 0.9322693 \\
\hline 25 & 0.7203572 & 0.6410306 & 0.7879527 & 39 & 0.9092975 & 0.8639835 & 0.9405539 \\
\hline 26 & 0.7394853 & 0.6714189 & 0.7977000 & 40 & 0.9169910 & 0.8701523 & 0.9479448 \\
\hline 27 & 0.7577450 & 0.6997189 & 0.8076407 & 41 & 0.9240864 & 0.8759761 & 0.9545035 \\
\hline 28 & 0.7751141 & 0.7256294 & 0.8179135 & 42 & 0.9306212 & 0.8814959 & 0.960299 \\
\hline 29 & 0.7915804 & 0.7488827 & 0.828679 & 43 & 0.936632 & 0.8867425 & 0.9654028 \\
\hline 30 & 0.807141 & 0.7692857 & 0.8400763 & 44 & 0.9421544 & 0.8917399 & 0.9698848 \\
\hline 31 & 0.8218014 & 0.7868013 & 0.8521355 & 45 & 0.9472227 & 0.8965071 & 0.9738117 \\
\hline
\end{tabular}

Table 13. Predicted Probabilities and Confidence Intervals for passing with grade of at least C-. 


\section{CONCLUSIONS}

All models in this analysis show that there is a significant positive relationship between IAD scores and final grades for students registering in the introductory statistics course Stat 1 at CSUS. The regression models indicate that on average an increase of one point in the IAD score increases the numerical final grade by 0.74 of a percentage point. The hierarchical linear model showed that this relationship of IAD scores and final grades is independent of the instructors.

While the linear regressions models produced confidence and prediction intervals that were too wide to be helpful, the logistic regression model did produce more narrow and informative confidence intervals. The confidence intervals indicate that for students with IAD scores of at least 32 , the maximum failing rate with a grade of $\mathrm{F}$, is on average $10 \%$. This percentage increases to $20 \%$ for students who need to pass the class with a grade of at least C-. The increasing bounds of the confidence intervals show that these failing rates increase as the IAD scores decrease. This model can then be used by the department to adjust the minimum IAD score based on its goals in terms of failing rates for Stat 1 .

Therefore, the results of this study supports the use of the IAD test as a tool to screen students registering in Stat 1. The IAD test is not necessarily testing students in statistical concepts, but it may indeed be measuring a level of abstract thinking required for success in Stat 1. IAD scores are not predictive of particular final grades in Stat 1, but they are useful in predicting the failing, and passing, rates in this course. Current efforts at CSUS and other universities to expedite students' time-to-degree may find this study useful in helping them decide the risks of enrolling underprepared students in Stat 1.

\section{ACKNOWLEDGEMENTS}

This work was supported by a "Bridges to the Baccalaureate" grant from the National Institute of General Medical Sciences (NIGMS), an institute of the National Institutes of Health (NIH). Grant No. GM056645.

We thank Roger Leezer $(1942-2011)$ for collecting and providing us with the data used in this study. 


\section{REFERENCES}

[1] Joseph, S. (2014) CSUS/UC Mathematics Diagnostic Testing Project, http://mdtp.ucsd.edu/history. shtml (accessed Nov 2014)

[2] Team, R. D. C. (2008) R: A language and environment for statistical computing, R Foundation for Statistical Computing, Austria, Vienna.

[3] Raudenbush, S., Bryk, A., Cheong, Y., and Congdon, R. (2004) HLM 7: Hierarchical linear and nonlinear modeling., Scientific Software International, Chicago, IL.

[4] Kleinbaum, D., Kupper, L., Muller, K., and Nizam, A. (1998) Straight-Line Regression Analysis 3rd ed., Duxbury Press, Pacific Grove, California.

[5] Snijders, T., and Bosker, R. (2012) The Random Intercept Model and the Hierarchical Linear Model, In Multilevel Analysis 2nd ed., pp 40 -93, SAGE Publications Inc., Thousand Oaks, California.

\section{ABOUT THE STUDENT AUTHORS}

Anthony Cooper is a mathematics major at California State University-Sacramento (CSUS). He is a non-traditional student that returned to college after working in a bank for 10 years and then serving children and families in an afterschool program for over 15 years. He is currently finishing his major and teaching credential studies at CSUS preparing for a career in education.

Justine Kirksey graduated from CSU, Sacramento Spring 2014 with her BA degree in Biology, minor in Mathematics. She is beginning her teaching credential program at National University in September to pursue her career as a mathematics and science high school teacher.

\section{PRESS SUMMARY}

The use of the intermediate algebra diagnostic (IAD) test as a screening tool for a statistics course (Stat 1) in the Department of Mathematics and Statistics at California State University Sacramento has been questioned by some faculty members and students at this university. The statistical analysis in this study shows that higher IAD scores are indeed related to higher final grades in Stat 1, even after adjusting for different instructors when using a sample of over 550 students who have taken this course. Inferences were also made in this study to predict passing grades and passing rates in Stat 1 when using IAD scores as predictors. 